\title{
Características Planimétricas y Estereológicas de los Nervios Palmar Lateral y Medial en Manos de Equinos con $y$ sin Síndrome Navicular
}

\author{
Planimetric and Stereological Characteristics of Lateral and Medial Palmar \\ Nerves in Hands of Equine with and without Navicular Syndrome \\ Paulo Salinas",*****; Francisco Rivas"; Saúl Figueroa*; Rodrigo Bañados $^{* * * * *}$ \& Carlos Sandoval $^{* * * * *}$
}

\begin{abstract}
SALINAS, P.; RIVAS, F.; FIGUEROA, S.; BAÑADOS, R. \& SANDOVAL, C. Características planimétricas y estereológicas de los nervios palmar lateral y medial en manos de equinos con y sin síndrome navicular. Int. J. Morphol., 33(4):1441-1447, 2015.

RESUMEN: El Síndrome Navicular (SN) es una de las patologías claudicógenas mas frecuentes en la práctica clínica equina. Se ha descrito, cierta susceptibilidad individual respecto a ésta presentación clínica, es decir, algunos equinos con SN claudican y otros, con similares hallazgos radiográficos en mano no lo hacen sugiriendo que existen diferencias cuantitativas, entre equinos con y sin SN, en las características de las fibras nerviosas de los nervios periféricos que inervan la mano. El objetivo del estudio fue describir las características morfológicas cuantitativas de los nervios palmar lateral y medial en equinos con SN. Se obtuvo manos mediante un muestreo dirigido y diagnosticadas por inspección visual y evaluación radiográfica. Se conformó dos grupos: "No afectadas" (n=6) y "Síndrome Navicular" ( $n=8)$. Para determinar diferencias cuantitativos entre ambos grupos se realizó: i) Estudio 1, evaluó las características morfológicas, planimétricas y estereológicas de los nervios palmar lateral y medial, ii) Estudio 2: evaluó la cantidad de axones mielinizados y no mielinizados. En ambos estudios, el análisis de los datos morfológicos, estereológicos y planimétricos, en general, no detectó diferencias significativas entre grupos. En conclusión, los resultados obtenidos en el estudio no entregan evidencia morfológica respecto a diferencias entre nervios palmares entre equinos con y sin SN.
\end{abstract}

PALABRAS CLAVE: Equino; Síndrome navicular; Estereología; Planimetría; Morfología; Nervio palmar lateral; Nervio palmar medial.

\section{INTRODUCCIÓN}

Las técnicas estereológicas (TE) se emplean cada vez con mayor frecuencia para la evaluación y descripción de enfermedades del sistema neuromuscular en medicina veterinaria y humana. Matiasek et al. (2008) sugieren el uso de éstas como una técnica complementaria durante la descripción histopatológica. En el estudio de los constituyentes de los nervios proporcionan evidencia cuantitativa, por tanto, entregan información sobre las características de la fibra nerviosa y una base para entender sus propiedades electrofisiológicas.

El Sindrome Navicular (SN) es un término utilizado para describir una presentación clínica asociada a dolor pal- mar y claudicación en equinos (Pool et al., 1989). Voute (2008) la define como una patología claudicógena bilateral, crónica, degenerativa y progresiva que compromete al HSD, la bolsa podotroclear, ligamentos y a las superficies adyacentes al tendón del músculo flexor digital profundo (tMFDP). Se caracteriza por un incremento en la presión que ejerce este último sobre el HSD y la articulación interfalángica distal (AIFDM) de la mano aumentando el contacto y tensión entre ellos. Respecto a la claudicación que presentan los equinos, Dyson et al. (2011) describen una susceptibilidad individual, i.e. algunos equinos con SN claudican y otros, con similares hallazgos radiográficos no lo hacen, sugiriendo que existen diferencias en la cantidad

\footnotetext{
Laboratorio de Anatomía Veterinaria, Escuela de Medicina Veterinaria, Facultad de Recursos Naturales y Medicina Veterinaria, Universidad Santo Tomás, Temuco, Chile.

** Programa de Doctorado en Ciencias Morfológicas, Facultad de Medicina, Universidad de La Frontera, Temuco, Chile.

*** Becario CONICYT.

***** Servicio de Veterinaria, Región de La Araucanía, Carabineros de Chile, Temuco, Chile.

****** Laboratorio I+D Veterquímica, Puerto Montt, Chile.
} 
de fibras nerviosas que inervan la mano o variabilidad en la cantidad de terminaciones nerviosas sensoriales presentes en los ligamentos sesamoídeos colaterales de la AIFDM. Bases de datos neuromorfométricas en la región de la mano en equino son escasas (Matiasek et al.) y no logran confirmar con certeza un rol preponderante de estas estructuras con la no-claudicación. Nuestro interés en la descripción estereológica de los nervios que inervan la mano y dedo equino se centra en entender posibles diferencias morfológicas cuantitativas que influirían sobre la presentación clínica de claudicación en equinos.

\section{MATERIAL Y MÉTODO}

Material biológico. Se utilizaron manos izquierdas de 14 equinos (Equus ferus caballus) destinados a sacrificio por razones no relacionadas con esta investigación (edad: $6 \pm 3$ años; peso promedio: $350 \pm 76 \mathrm{~kg}$ ) y que presentaron características externas sugerentes de SN tales como: baja angulación del dedo, paredes largas y concavidad en la cara solear de la úngula, y manos sin despalmes correctivos (Stashak, 2004). El procesamiento de las manos previo a la etapa de diagnóstico se realizó según lo descrito previamente en Salinas et al. (2014a, 2014b).

Diagnóstico de Síndrome Navicular y grupos. Se realizó mediante una evaluación morfológica y radiográfica. Los hallazgos radiográficos y criterios utilizados para diagnosticar SN en este estudio fueron los utilizados por Salinas $e t$ al. (2014a, 2014b). Se conformó los siguientes grupos: No Afectadas $(n=6)$ y Síndrome Navicular $(n=8)$.

Procesamiento de muestras. Fueron disecadas y extraídas secciones $(1 \mathrm{~cm})$ de tejido nervioso de NPL y NPM y sus ramos (ramo dorsal del nervio digital palmar lateral: rdNDPL; ramo dorsal del nervio digital palmar medial: rdNDPM; nervio digital palmar lateral: NDPL; nervio digital palmar medial: NDPM). Cada sección fue rotulada y fijada en formaldehido $10 \%$, posicionada longitudinalmente y embebida en parafina sólida. Se obtuvo secciones transversales de $5 \mu \mathrm{m}$ de grosor las que fueron teñidos con tinción convencional de hematoxilina-eosina. Las observaciones se realizaron en un microscopio Leica ${ }^{\circledR}$ DM750, provisto con objetivos $2 \mathrm{X}, 10 \mathrm{X}, 40 \mathrm{X}$ y 100X, y equipado con cámara digital Leica ${ }^{\circledR}$ ICC50 HD, que permitió la proyección de campos microscópicos en un sistema de prueba planimétrico y estereológico de área conocida. Respecto a la disección de los nervios y la medicion de las variables morfológicas, planimétricas y estereológicas, éstas fueron realizadas por dos evaluadores con el objetivo de disminuir la subjetividad y el sesgo de evaluador.
Estudios. Se realizaron dos estudios (Fig. 1). El Estudio 1 evaluó la morfología, estereología y planimetría de cada sección nerviosa obtenida de NPL y NPM y sus ramos. El estudio morfológico evaluó en secciones nerviosas transversales: i) el área ii) y contabilizó el número de fascículos. El estudio estereológico evaluó (10X) parámetros tales como: i) densidad de superficie $\left(\mathrm{S}_{\mathrm{v}}\right)$ y ii) densidad de volumen $\left(\mathrm{V}_{\mathrm{v}}\right)$ de fascículos nerviosos (10X). El estudio planimétrico evaluó la proporción de área (\%) de i) epineuro superficial y profundo (Epineurium superficiale et profundum), ii) fascículo nervioso y perineuro (Perineurium) y iii) Vasa-nervorum y fueron determinados mediante el análisis de imágenes digitales de secciones nerviosas transversales, discriminando entre las superficies de los diferentes componentes de interés montando sobre las imágenes un sistema planimétrico de puntos de distancia calibrada y área conocida (Sistema Test). Se analizó 3 campos por cada sección. En el Estudio 2 se aplicó técnicas estereológicas en secciones transversales de fascículos de NPL y NPM y sus ramos para evaluar la cantidad de axones mielinizados (Neurofibra myelinata) y nomielinizados (Neurofibra nonmyelinata) en ambos grupos. Se analizó tres campos (40X) por cada sección histológica y se midió la densidad numérica por área $\left(\mathrm{N}_{\mathrm{A}}\right)$ de axones mielinizados y no-mielinizados. Los criterios utilizados para identificar los tipos de axones fueron descritos por Dellmann \& Eurell (1998). Para determinar el $\mathrm{N}_{\mathrm{A}}$ de axones nomielinizados se utilizó la formula propuesta por Mandarim de Lacerda (1995). Primero se calculó el $\mathrm{N}_{\mathrm{A}}$ de los "complejos axón no-mielinizado - neurolemnocito" y posteriormente se aplicó un factor de conversión. Este útimo fue obtenido mediante el análisis al azar de 8 de 16 cuadrantes sobrepuestos a cada imagen digital de un fascículo y en los cuales se promedió el número de axones no-mielinizados por cada neurolemnocito (neurolemmocytus). Las evaluaciones planimétricas y estereológicas consideraron el factor de compresión (Dorph-Petersen et al., 2001) y se realizó según lo descrito por Baddeley et al. (1986) y Gundersen et al. (1988) con el test multipropósito $\mathrm{M}_{36}$ y el software STEPanizer ${ }^{\circledR}$ Stereological tool, version 1.0 (Fig. 2; Tschanz et al., 2011).

Análisis estadístico. El estudio fue de tipo descriptivo, transversal e inferencial. Los datos cuantitativos se ordenaron en tablas Excel (Microsoft ${ }^{\circledR}$ Office $^{\circledR} 2011$ ) y expresados como promedio $(\mathrm{X}) \pm$ desviación estándar (DE). Para detectar normalidad en los datos se aplicó el Test D'Agostino-Person. Para observar diferencias estadísticas significativas entre grupos se utilizó el Test paramétrico de Student. Los datos fueron transformados logarítmicamente cuando ambos grupos no coincidieron respecto a demostrar una misma distribución. La significancia estadística para todas las pruebas fue establecida para $\mathrm{p}<0,05$. El análisis de los datos se realizó con el software Prisma ${ }^{\circledR}$ 6.0 para MAC OS X (GraphPad Software, San Diego CA). 


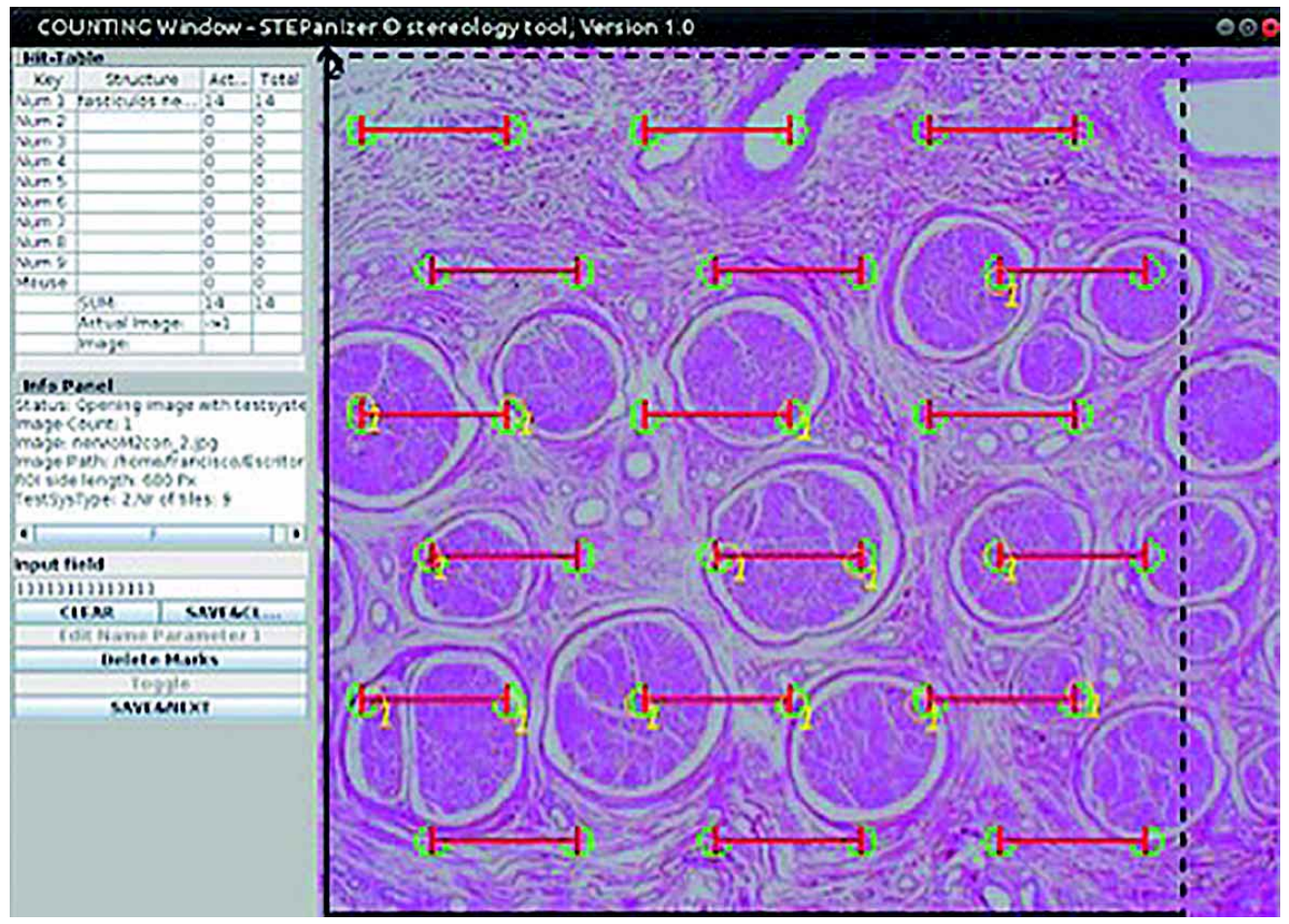

Fig. 1. Ejemplo de interfaz de Sistema Test ( S T E P a n i z e r ${ }^{\circ}$ stereological tool, Version 1.0), utilizado en nervio palmar lateral y nervio palmar medial y sus ramos para determinar densidad de volumen (VV) de fascículos nerviosos. La totalidad de los elementos que componen los sistemas de prueba como marcos, líneas continuas, líneas discontinuas, puntos y áreas son de dimensiones conocidas.

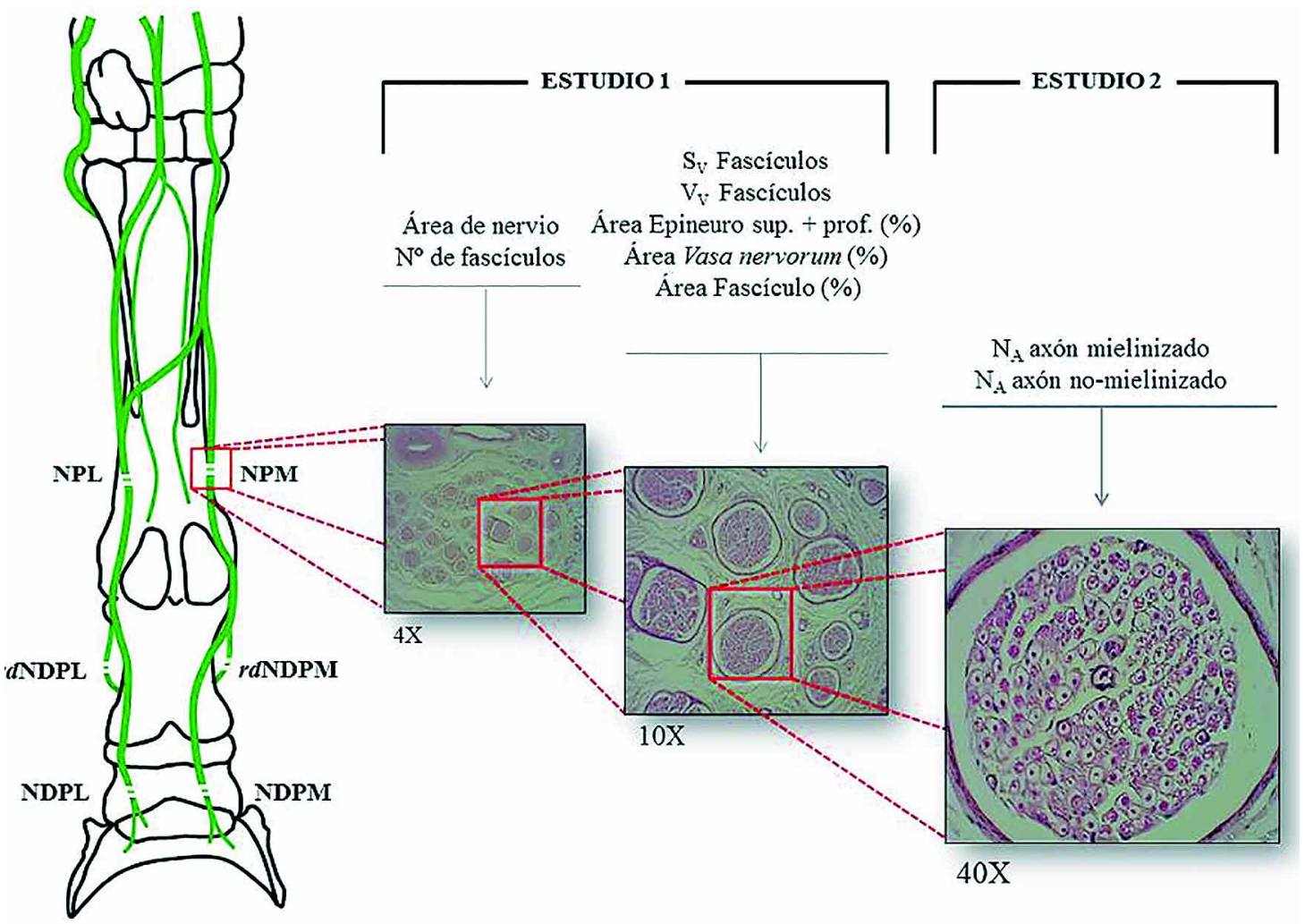

Fig. 2. Diseño experimental utilizado en el Estudio 1 y 2. Esquema de la inervación de mano equina izquierda, vista palmar. Señala el sitio de obtención, y posterior sección y tinción con hematoxilina eosina convencional. Nervio palmar lateral: NPL; nervio palmar medial: NPM; ramo dorsal del nervio digital palmar lateral: rdNDPL; ramo dorsal del nervio digital palmar medial: rdNDPM; nervio digital palmar lateral: NDPL; nervio digital palmar medial: NDPM. 


\section{RESULTADOS}

La Tabla I muestra parámetros morfológicos y estereológicos evaluados en el NPL y NPM y sus ramos en manos de equinos con Síndrome Navicular (SN) y No Afectadas (NA). Describe resultados obtenidos en el Estudio 1, tales como promedios de área de sección nerviosa, y cantidad, $\mathrm{S}_{\mathrm{v}} \mathrm{y} \mathrm{V}_{\mathrm{V}}$ de fascículos nerviosos en secciones transversales. El análisis estadístico no detectó diferencias significativas entre grupos en ninguno de los nervios y ramos evaluados. Además, muestra parámetros estereológicos evaluados en el Estudio 2 tales como $\mathrm{N}_{\mathrm{A}}$ de axones mielinizados y no-mielinizados en fascículos. El análisis estadístico detectó diferencias significativas entre grupos en el NPL $(\mathrm{p}=0,0411)$ y NDPL $(\mathrm{p}=0,0022)$. Respecto al estudio planimétrico, la Figura 3 muestra resultados planimétricos y la proporción de área de epineuro superficial y profundo, fascículos nerviosos y perineuro, y vasa-nervorum en NPL y NPM y sus respectivos ramos, en manos de equinos con SN y NA, y la Tabla II muestra la magnitud de las probables diferencias (valor-p) en la proporción de tejidos que constituyen el NPL y NPM, y sus respectivos ramos, en manos de equinos con SN y NA. El análisis estadístico detectó diferencias significativas entre grupos en el área de fascículos y perineuro del $\operatorname{rdNDPM~}(\mathrm{p}=0,0308)$.

\section{DISCUSIÓN}

Se estudió las características morfológicas planimétricas y estereológicas de los NPL/M y sus ramos en equinos con SN. Se realizó dos estudios morfológicos. El Estudio 1 evaluó la morfología, planimetría y estereología de los componentes del nervio periférico y el Estudio 2 evaluó el número de axones mielinizados y no-mielinizados en los fascículos nerviosos de ambos grupos de estudio.

Respecto a las etapas de obtención, diagnóstico de manos y conformación de grupos fueron realizadas según los criterios descritos en la literatura especializada (Stashak; Bentley et al., 2007; Floyd \& Mansmann, 2009; Waguespack \& Hanson, 2010; Dyson et al.; Komosa et al., 2013). La mayoría de los signos macroscópicos observados en manos y los hallazgos radiográficos evidenciaron la existencia de procesos patológicos en el HSD que lo afectan como respuesta a los apremios mecánicos debido a altas exigencias, todos asociados al SN (Komosa et al.). Por lo tanto, creemos que los criterios utilizados para realizar la conformación de grupos y posteriormente el diagnóstico, son representativos de manos equinas con SN y desde un punto de vista clínico son los utilizados con mayor frecuencia. Respecto a la disección de los nervios y las técnicas morfológicas, planimétricas y estereológicas realizados en este estudio, éstas fueron realizadas por dos evaluadores con el propósito de disminuir la subjetividad y sesgo de evaluador.

Respecto al área de sección nerviosa en NPL y NPM y sus ramos no se detectó diferencias significativas entre grupos. Creemos que esto es atribuido a la homogeneidad de las manos (equinos mestizos y de peso promedio $350 \mathrm{~kg}$.). Cabe señalar que Matiasek et al., midieron áreas de sección de nervio periférico en equinos, tomando como referencia al nervio fibular común y describieron resultados similares a las reportadas en nuestro estudio en NPL y NPM.

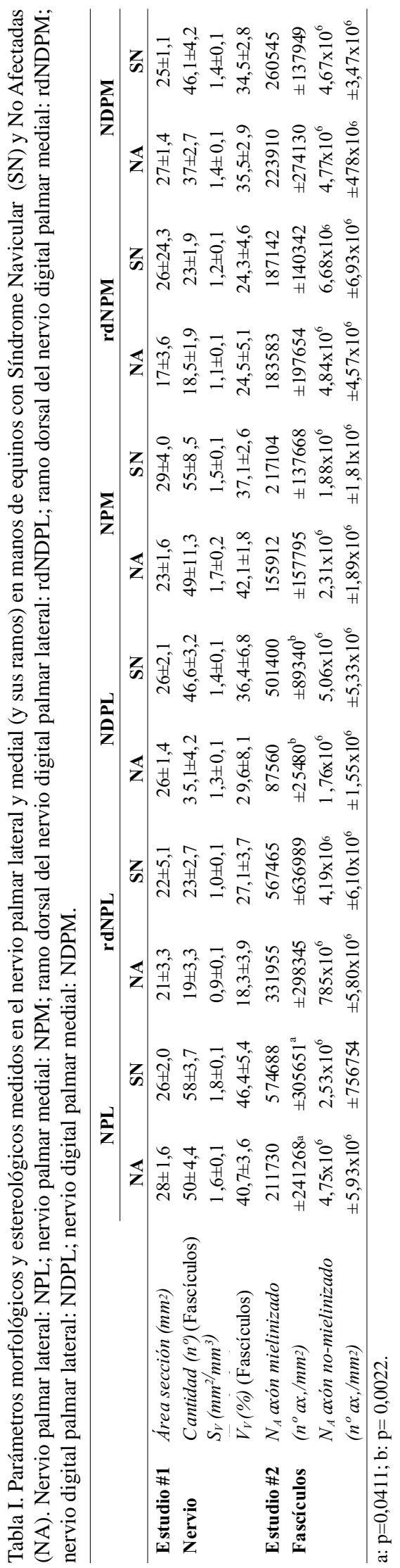




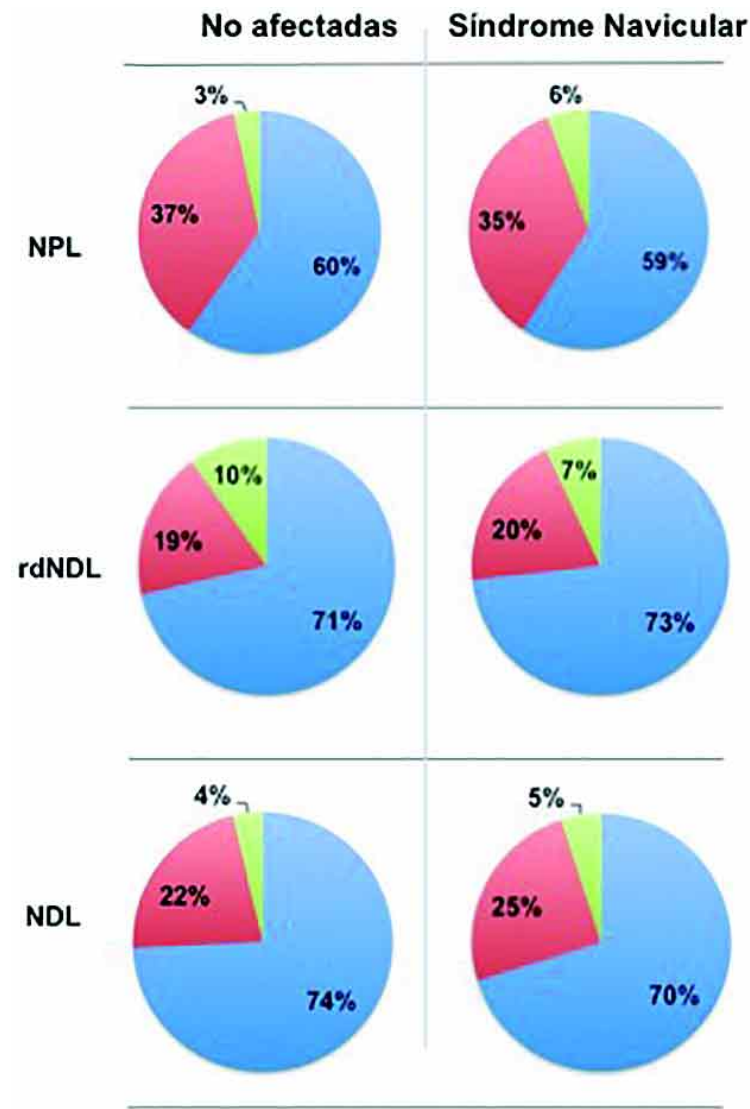

EPINEURO SUP. \& PROF.

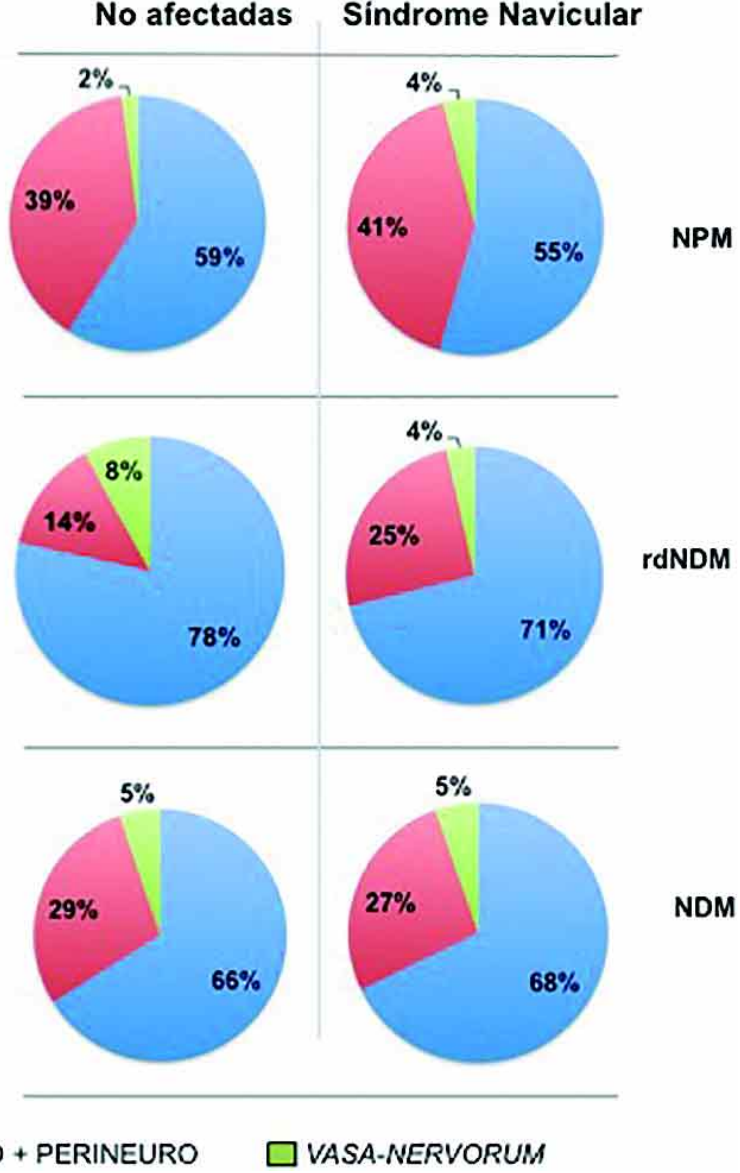

FASCICULO + PERINEURO
VASA-NERVORUM

Fig. 3. Estudio planimétrico en manos de equinos con Síndrome Navicular y No Afectadas. Proporción de áreas (\%) de: i) epineuro superficial y profundo, ii) fascículos nerviosos y perineuro y iii) vasa-nervorum por sección nerviosa de: nervio palmar lateral (NPL), nervio palmar medial (NPM), ramo dorsal del nervio digital palmar lateral (rdNDPL), ramo dorsal del nervio digital palmar medial (rdNDPM), nervio digital palmar lateral (NDPL) y nervio digital palmar medial (NDPM).

Tabla II. Magnitud de las probables diferencias (valor-p) en la proporción de tejidos que constituyen el nervio palmar lateral y medial (y sus ramos) en manos de equinos con Síndrome Navicular vs. No Afectadas. Nervio palmar lateral: NPL; nervio palmar medial: NPM; ramo dorsal del nervio digital palmar lateral: rdNDPL; ramo dorsal del nervio digital palmar medial: rdNDPM; nervio digit al palmar lateral: NDPL; nervio digital palmar medial: NDPM.

\begin{tabular}{lcccccc}
\hline & NPL & rdNDPL & NDPL & NPM & rdNDPM & NDPM \\
\hline Epineuro Sup, \& Prof, & 0,5741 & 0,8639 & 0,7491 & 0,5984 & 0,4662 & 0,6354 \\
Fas ciculo + Perineuro & 0,7083 & 0,965 & 0,597 & 0,2262 & $0,0308^{*}$ & 0,5935 \\
Vasa-nervorum & 0,3250 & 0,572 & 0,631 & 0,3431 & 0,2481 & 0,8988 \\
\hline
\end{tabular}

Respecto a la cantidad de fascículos nerviosos en NPL y NPM y sus ramos no se detectó diferencias significativas entre grupos en ninguna de las evaluaciones realizadas. Sin embargo, en un mismo grupo observamos desde proximal a distal lo que Sunderland et al. (1959) describe como "formaciones plexurales", i.e. los fascículos nerviosos durante todo su trayecto hacia distal se unen y dividen en reiteradas ocasiones. El aumento de bifurcaciones es común en los nervios ubicados en articulaciones de gran movilidad (v.g. articulación escápulo-humeral), otorgando una mayor elasticidad, flexibilidad y capacidad de elongación al tejido nervioso. Respecto a la $\mathrm{S}_{\mathrm{v}}$ y $\mathrm{V}_{\mathrm{v}}$ de fascículos en NPL y NPM y sus ramos no se detectó diferencias significativas entre grupos. Observamos en manos de equinos con SN que la superficie que ocupan los fascículos por unidad de volumen $\left(\mathrm{mm}^{2} /\right.$ $\mathrm{mm}^{3}$ ) y proporción de volumen (\%) es similar a lo observa- 
do en grupo NA. Las no-diferencias detectadas entre grupos indican que respecto a la superficie y volumen que ocupan los fascículos en las secciones nerviosas evaluadas, el SN no tendría un rol relevante respecto a variaciones evidentes.

Respecto al estudio planimétrico, se evaluó el área del tejido que constituyen las secciones del NPL y NPM y sus respectivos ramos en manos de equinos con SN y NA. Cada medición consistió en la evaluación de la proporción de área que constituían: epineuro superficial y profundo, fascículos y perineuro, y vasa-nervorum respecto al total de superficie de la sección transversal. Posteriormente se realizó la descripción de las magnitudes de las probables diferencias (valor-p) en la proporción de tejidos que constituyen los nervios evaluados. Nuestros resultados evidencian diferencias significativas en la proporción de área fascículos y perineuro en las secciones obtenidas de rdNDPM evaluadas entre grupos.

Respecto a $\mathrm{N}_{\mathrm{A}}$ (densidad numérica por área) de axones mielinizados y no-mielinizados medidos en NPL y NPM y sus respectivos ramos, por lo general, no se encontró diferencias significativas entre grupos en ninguna de las evaluaciones realizadas. Sin embargo, se encontró diferencias significativas entre grupos en la cantidad de axones mielinizados en NPL $(p=0,0411)$ y NDPL ( $p=0,0022)$. Los resultados obtenidos son similares a lo descrito en nervios equinos reportados con anterioridad tales como: nervios fibular común y profundo, y nervio laríngeo recurrente (Slocombe et al., 1992), y nervios accesorio y nervio fibular común (Matiasek et al.). Respecto a la diferencia observada en la cantidad de axones mielinizados y no-mielinizados por área, ésta pueden atribuirse a: i) un proceso inflamatorio localizado en la región evaluada, propio de un proceso degenerativo nervioso o de reparación (brotes axonales) que podría aumentar o disminuir la variable medida (Rodríguez, 2009) y ii) al azar en la obtención de la muestra (Manterola \& Pineda, 2008), ya que los muestreos realizados pueden haber seleccionado azarosamente a las manos de equinos que presentaron diferencias estereológicas en la composición de las fibras nerviosas (sesgo de selección).

Finalmente, aún cuando existen reportes que describen cambios macro y microscópicos es importante considerar que la presentación de SN es consecuencia de una amplia cantidad de factores que pueden generar variaciones morfológicas en los componentes del sistema podotroclear, tales como el HSD y en la matriz ósea de este último, sin embargo no hay evidencia experimental de variaciones en las características morfológicas de nervios que inervan la mano. Creemos que el desarrollo de estudios longitudinales que complementen los resultados obtenidos y que además demuestren variaciones que puedan ser atribuidas al SN tales como estudios de receptores en ligamentos propios del sistema podotroclear, en la velocidad de conducción del impulso nervioso, en la vaina de mielina de los nervios, en el tejido del tMFDP y en bolsa podotroclear - aportarán en la discusión de la implementación de estrategias preventivas y terapias de recuperación en el equino.

SALINAS, P.; RIVAS, F.; FIGUEROA, S.; BAÑADOS, R. \& SANDOVAL, C. Planimetric and stereological characteristics of lateral and medial palmar nerves in hands of equine with and without navicular syndrome. Int. J. Morphol., 33(4):1441-1447, 2015.

SUMMARY: Navicular Syndrome (NS) is one of the most frequent claudicogen pathologies in the equine clinical practice. Certain individual susceptibility has been described in this clinical presentation, that is, some horses with SN halt, while others with similar radiographic findings in hand do not, suggesting that there are quantitative differences, between horses with and without clinical presentation of SN, on the characteristics of the nerve fibers of the peripheral nerves of the hand. The aim of this study was to describe the morphologic quantitative characteristics of the lateral and medial palmar in horses with SN nerves. Hands were obtained by sampling directed and diagnosed by visual inspection and radiographic evaluation. Two groups were formed: "Not affected" $(n=6)$ and "Navicular Syndrome" $(n=8)$. To determine quantitative differences between two groups we performed: i) Study 1: evaluated the morphological, planimetric and stereological lateral and medial palmar nerves, ii) Study 2: evaluated the number of unmyelinated and myelinated axons. In both studies, analysis of stereological, morphological and planimetric data generally detected no significant differences between groups. In conclusion, the results of the study do not provide morphological evidence for differences between palmar nerves between horses with and without SN. nerve.

KEY WORDS: Equine; Navicular syndrome; Stereology; Planimetry; Morphology; Palmar lateral nerve; Medial palmar

\section{REFERENCIAS BILBIOGRÁFICAS}

Baddeley, A. J.; Gundersen, H. J. \& Cruz-Orive, L. M. Estimation of surface area from vertical sections. J. Microsc., 142(Pt. 3):259-76, 1986.
Bentley, V. A.; Sample, S. J.; Livesey, M. A.; Scollay, M. C.; Radtke, C. L.; Frank, J. D.; Kalscheur, V. L. \& Muir, P. Morphologic changes associated with functional adaptation of the navicular bone of horses. J. Anat., 211(5):662-72, 2007. 
Dellmann, H. D. \& Eurell, J. Textbook of Veterinary Histology. 5th ed. Philadelphia, Lippincott Williams \& Wilkins, 1998.

Dorph-Petersen, K. A.; Nyengaard, J. R. \& Gundersen, H. J. Tissue shrinkage and unbiased stereological estimation of particle number and size. J. Microsc., 204(Pt. 3):232-46, 2001 .

Dyson, S.; Murray, R.; Schramme, M. \& Blunden, T. Current concepts of navicular disease. Equine Vet. Educ., 23(1):27$39,2011$.

Floyd, A. E. \& Mansmann, R. A. Podiatría Equina. Buenos Aires, Intermédica, 2009. pp.4-9, 30-1,

Gundersen, H. J.; Bendtsen, T. F.; Korbo, L.; Marcussen, N.; Møller, A.; Nielsen, K.; Nyengaard, J. R.; Pakkenberg, B.; Sørensen, F. B.; Vesterby, A. \& West, M. J. Some new, simple and efficient stereological methods and their use in pathological research and diagnosis. APMIS, 96(5):379-94, 1988.

Komosa, M.; Purzyc, H. \& Frackowiak, H. Changes in navicular bone (os sesamoideum distale) shape in horses as a result of pathological alterations. Folia Biol. (Krakow), 61(1-2):1-10, 2013.

Mandarim-de-Lacerda, C. A. Metodos Quantitativos em Morfologia. Rio de Janeiro, Editora da Universidade do Estado do Rio de Janeiro, 1995. pp.1-20, 75-85.

Manterola D. C.; Pineda N., V. \& Grupo MINCIR. El valor de "p" y la "significación estadística". Aspectos generales y su valor en la práctica clínica. Rev. Chil. Cir., 60(1):86-9, 2008.

Matiasek, K.; Gais, P.; Rodenacker, K.; Jütting, U.; Tanck, J. J. $\&$ Schmahl, W. Stereological characteristics of the equine accessory nerve. Anat. Histol. Embryol., 37(3):205-13, 2008.

Pool, R. R.; Meagher, D. M. \& Stover, S. M. Pathophysiology of navicular syndrome. Vet. Clin. North Am. Equine Pract., 5(1):109-29, 1989.

Rodríguez, R. La biopsia en el diagnóstico de la enfermedad pediátrica. Acta Pediatr. Mex., 30(3):175-81, 2009.

Salinas, P.; Figueroa, S.; Carrasco, C. \& Bañados, R. Morphometry, planimetry and stereology in the distal sesamoid bone in equine foot with and without navicular syndrome. Int. J. Morphol., 32(1):357-63, 2014a.

Salinas, P.; Figueroa Vergara, S. A. \& Bañados Fernández, R. Histochemical study of the distribution of collagen fibers on distal sesamoid bone in equine with and without navicular syndrome. Int. J. Morphol., 32(4):1266-70, 2014 b.

Slocombe, R. F.; Huntington, P. J.; Friend, S. C.; Jeffcott, L. B.; Luff, A. R. \& Finkelstein, D. K. Pathological aspects of Australian Stringhalt. Equine Vet. J., 24(3):174-83, 1992.
Stashak, T. Adams' Lameness in horses. $4^{\text {th }}$ ed. Philadelphia, Lea \& Febiger, 2004. pp.113-7, 706-22.

Sunderland, S.; Marshall, R. D. \& Swaney, W. E. The intraneural topography of the circumflex, musculocutaneous and obturator nerves. Brain, 82(1):116-29, 1959.

Tschanz, S. A.; Burri, P. H. \& Weibel, E. R. A simple tool for stereological assessment of digital images: the STEPanizer. $J$. Microsc., 243(1):47-59, 2011.

Voute, L. What can radiology tell us about palmar foot pain? Liverpool, Proceedings of the Proceedings of the $47^{\text {th }}$ British Equine Veterinary Association Congress, 2008. pp.30-2.

Waguespack, R. \& Hanson, R. R. Navicular syndrome in equine patients anatomy, causes, and diagnosis. Compend. Contin. Educ. Vet., 32(12):E7, 2010.

Dirección para correspondencia

Paulo Salinas Pérez, MV, MSc.

Laboratorio de Anatomía Veterinaria

Escuela de Medicina Veterinaria

Universidad Santo Tomás

Manuel Rodríguez 060

Temuco

CHILE

Email: psalinas@santotomas.cl

Recibido : 02-04-2015

Aceptado: 23-09-2015 